\title{
Oral Dysbiosis - A Consequence of Microbial Shift and A Path to Periodontal Disease
}

\author{
Samriti katoch* \\ Department of Periodontology, Seema Dental College and Hospital, Rishikesh, Uttarakhand, India \\ *Corresponding Author: Samriti Katoch, Department of Periodontics, Seema Dental College and Hospital, Rishikesh, Uttarakhand, India.
}

Received: September 17, 2019; Published: October 23, 2019

DOI: 10.31080/ASDS.2019.03.0674

\section{Abstract}

The oral cavity provides the habitat to wide variety of microorganisms. These microorganisms live as commensals and do not cause any harm to the host, but as the conditions change such as poor oral hygiene practises, more sugar intake, immunocompromised disease these microorganisms are perfectly capable of causing the disease. This is referred to as oral dysbiosis that is overpopulation of the pathogenic species and periodontal disease is a classical example of such a condition.

Keywords: Oral Dysbiosis; Commensals; Periodontal Disease

\section{Introduction}

The oral cavity provides with a wide variety of surfaces, each of which is coated mostly with bacteria and various other microorganisms. The overgrowth of these microorganisms may lead to caries or periodontal disease [1].

The host-bacterial relationships can be categorised as present:

- Symbiosis: It has been derived from a greek word which means living together of unlike microorganisms. It could further be of following types:

- Mutualism: Both the host and the bacteria derives benefit from each other.

- Commensalism: The bacteria derives the benefit without harming the host.

- Parasitism: The bacteria derives the benefit at the expense of the host

- Dysbiosis: The relative increase in the number of pathogens and decrease in the number of beneficial species resulting in microbial shift.

\section{Pathobionts}

These microorganisms live as commensals but as the conditions change they are perfectly capable of causing disease in susceptible host [2]. In healthy individuals with good oral hygiene habits and the right dietary habits, the host and the microorganisms live in a symbiotic relationship and prevents further colonization by foreign pathogens.
National institute of health (NIH) has launched a project named Human Microbiome Project that primarily aims to characterize the normal microflora of five regions of human body namely:

- Oral cavity

- Nasal passage

- GIT

- Genito-urinary tract

There are over 700 bacterial species residing in the oral cavity. However it is very difficult to characterize the normal oral microbial flora due to varied vast diversity in microflora between individuals, between different sites in the same individual and because of different dietary and oral hygiene habits [3].

Disruption of the balance in the ecosystem of the oral cavity leads to various dental diseases. This change is referred to as microbial shift. Susceptibility of each individual to the microbial shift varies, some are more tolerant while others are more susceptible to disease development. The microbial shift in the oral cavity leads to following changes.

Periodontal disease as we know are multifactorial, polymicrobial infectious, inflammatory type of disease with different pathogenic mechanisms and different clinical presentations. In periodontitis one germ one disease concept holds not much importance rather the entire microbial community is considered pathogenic [4]. Lack of beneficial species leads to colonization of sites by pathogenic flora and thereby contributing to disease. The change in the 


\begin{tabular}{|lll|}
\hline Gram positive & $\longmapsto$ & Gram negative \\
\hline Facultative anerobes & $\rightleftarrows$ & Obligate anerobes \\
\hline Cocci & $\rightleftarrows$ & Rods \\
\hline Non motile & $\rightleftarrows$ & Motile \\
\hline Fermentative & $\rightleftarrows$ Proteolytic \\
\hline
\end{tabular}

Table1: Microbial shift in the oral cavity.

microflora in periodontitis consists primarily of gram positive aerobes to gram negative anerobes.

Periodontitis is often called as a prototype disease associated with dysbiosis [5]. There is an extended period of time required for the development of dysbiosis eventually changing the symbiotic host relationship to a more pathogenic one. The first microbial complex that predominates in diseases states is the orange complex i.e) Prevotella intermedia and Fusobacterium nucleatum followed by the predominance of the red complex microorganisms i.e) Porphyromonas gingival is, Tinnerello forsythia, and Treponema denticola [6].

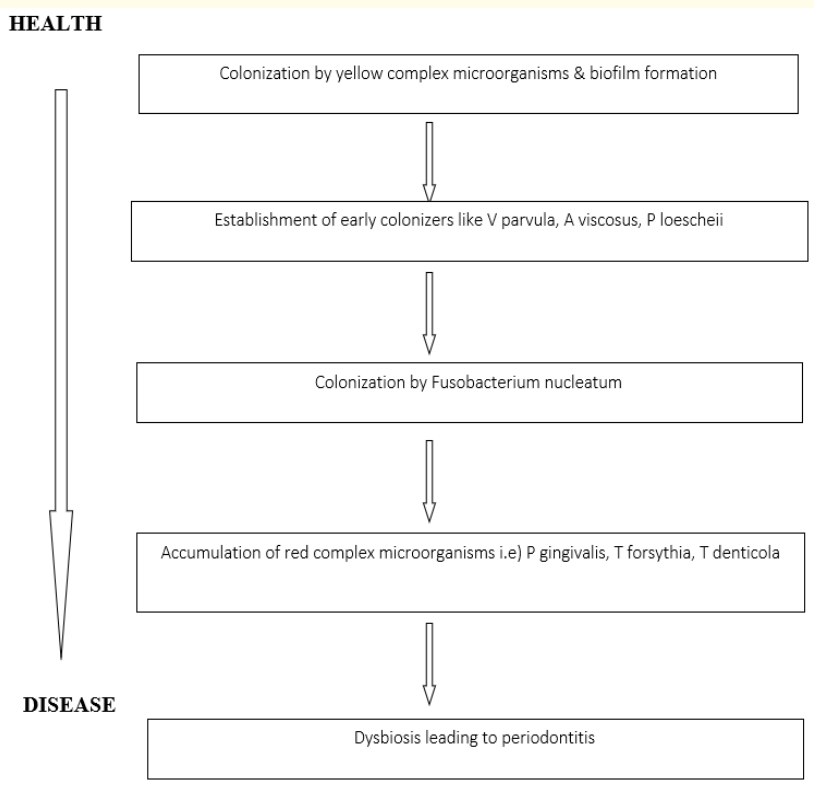

Figure 1: Dysbiosis in the development of periodontitis.

\section{Reconsidering Koch's Postulates}

Koch's Postulates cannot be very well applied to periodontal pathogens because of two main reasons. First, different periodontal pathogens show different clinical presentations and second, similar clinical presentations may be shown by different periodontal pathogens. Moreover, the pathogenic mechanisms of periodontitis are modified by various environmental, genetic and systemic risk factors that further complicates the application of Koch's Postulates [7].

\section{Bacterial - bacterial and host - bacterial interactions}

Cooperative and competitive metabolic and physiologic interactions among oral bacteria influence dysbiosis largely. Cooperative interactions involve when waste of one species serves as food for another. However bacteria also compete with each other for common sources required for growth. Furthermore some bacteria are even capable of releasing certain toxins that inhibit the growth of neighbouring bacteria. Aggregatibacter actinomycetemcomitans is one such bacteria that releases bacteriocin named as Actinobacillin. Bacteriocins from different bacteria possess different specificity against oral bacteria [2].

Accumulating evidence supports the "keystone-pathogen hypothesis" in which colonization of keystone bacteria such as Porphyromonas gingival is triggers dysbiosis. individual oral pathobionts that accumulate during dysbiosis play a critical and specific role in periodontitis development. oral dysbiosis, or a shift from beneficial symbiotic bacteria to pathogenic bacteria, is at least partially responsible for the development of periodontitis. However, despite great advances in our knowledge of the underlying microbial basis of this disease, the fact still remains that periodontitis has multiple etiologist which have yet to be fully understood. Thus, while a microbial shift is known to play a significant role in the development of periodontitis, genetic, immunologic, and environmental factors must also be investigated in order for clinicians and researchers to fully understand disease progression.

\section{Conflict of Interest}

Nil.

\section{Bibliography}

1. Albander JM., et al. "Destructive periodontal disease in adults 30 years of age and older in united states1988-1994". Journal of Periodontology 70.1 (1999) :13-29.

2. Jiao Y., et al. "The Role of Oral Pathobionts in Dysbiosis during Periodontitis Development". Journal OF Dental Research 93.6 (2014): 539-546.

3. Aas JA., et al. "Defining the normal bacterial flora of the oral cavity". Jounal of Clinical Microbiology 43 (2005): 5721-5732.

4. Siqueira JF., et al. "Community as the unit of pathogenicity: an emerging concept as to the microbial pathogenesis of apical periodontitis". Oral Surgery Oral Medicine Oral Pathology Oral Radiology and Endodontics 107 (2009) :870-878.

5. Socransky SS., et al. "Microbial complexes in subgingival plaque". Jounal of Clinical Periodontology 25(1998):134-144. 
6. Socransky SS., et al. "Periodontal microbial ecology". Periodontology 200038 (2005) :135-187.

7. Alex B., et al. "Microbial Shift and Periodontitis". Periodontology 2000 55.1(2011): 36-47.

Volume 3 Issue 11 November 2019

(C) All rights are reserved by Samriti katoch. 